\title{
Asymptotic estimates for the best uniform approximations of classes of convolution of periodic functions of high smoothness *
}

\author{
A.S. Serdyuk and I.V. Sokolenko \\ Dedicated to the memory of \\ Professor S.B. Stechkin and Professor S.A. Telyakovskii.
}

Abstract. We find two-sides estimates for the best uniform approximations of classes of convolutions of $2 \pi$-periodic functions from unit ball of the space $L_{p}, 1 \leq p<\infty$, with fixed kernels, modules of Fourier coefficients of which satisfy the condition $\sum_{k=n+1}^{\infty} \psi(k)<\psi(n)$. In the case of $\sum_{k=n+1}^{\infty} \psi(k)=o(1) \psi(n)$ the obtained estimates become the asymptotic equalities.

MSC 2010. $42 \mathrm{~A} 10$

Key words. Best approximation, Fourier sum, Weyl-Nagy class, $(\psi, \bar{\beta})$-integral, asymptotic equality, Kolmogorov-Nikol'skii problem.

Let $L_{p}, 1 \leq p<\infty$, be the space of $2 \pi$-periodic functions $f$ summable to the power $p$ on $[-\pi, \pi)$, in which the norm is given by the formula

$$
\|f\|_{L_{p}}=\|f\|_{p}=\left(\int_{-\pi}^{\pi}|f(t)|^{p} d t\right)^{1 / p},
$$

$L_{\infty}$ be the space of measurable and essentially bounded $2 \pi$-periodic functions $f$ with the norm

$$
\|f\|_{L_{\infty}}=\|f\|_{\infty}=\underset{t}{\operatorname{ess} \sup }|f(t)|,
$$

*This work was partially supported by the Grant H2020-MSCA-RISE-2019, project number 873071 (SOMPATY: Spectral Optimization: From Mathematics to Physics and Advanced Technology). 
and $C$ be the space of continuous $2 \pi$-periodic functions $f$, in which the norm is defined by the equality

$$
\|f\|_{C}=\max _{t}|f(t)|
$$

Denote by $C_{\bar{\beta}, p}^{\psi}, 1 \leq p \leq \infty$, the set of all $2 \pi$-periodic functions $f$, representable as convolution

$$
\begin{gathered}
f(x)=\frac{a_{0}}{2}+\frac{1}{\pi} \int_{-\pi}^{\pi} \varphi(x-t) \Psi_{\bar{\beta}}(t) d t, \quad a_{0} \in \mathbb{R}, \quad \varphi \in B_{p}^{0}, \\
B_{p}^{0}=\left\{\varphi \in L_{p}:\|\varphi\|_{p} \leq 1, \varphi \perp 1\right\},
\end{gathered}
$$

with a fixed generated kernel $\Psi_{\bar{\beta}} \in L_{p^{\prime}}, 1 / p+1 / p^{\prime}=1$, the Fourier series of which has the form

$$
S\left[\Psi_{\bar{\beta}}\right](t)=\sum_{k=1}^{\infty} \psi(k) \cos \left(k t-\frac{\beta_{k} \pi}{2}\right), \quad \beta_{k} \in \mathbb{R}, \quad \psi(k) \geq 0 .
$$

A function $f$ in the representation (1) is called $(\psi, \bar{\beta})$-integral of the function $\varphi$ and is denoted by $\mathcal{J}_{\bar{\beta}}^{\psi} \varphi\left(f=\mathcal{J}_{\bar{\beta}}^{\psi} \varphi\right)$. If $\psi(k) \neq 0, k \in \mathbb{N}$, then the function $\varphi$ in the representation $(1)$ is called $(\psi, \bar{\beta})$-derivative of the function $f$ is denoted by $f_{\bar{\beta}}^{\psi}\left(\varphi=f_{\bar{\beta}}^{\psi}\right)$. The concepts of $(\psi, \bar{\beta})$-integral and $(\psi, \bar{\beta})$-derivative was introduced by Stepanets (see, e.g., [31,32]). Since $\varphi \in L_{p}$ and $\Psi_{\bar{\beta}} \in L_{p^{\prime}}$, then (see. [32, Proposition 3.9.2.]) the function $f$ of the form (1) is a continuous function, i.e. $C_{\bar{\beta}, p}^{\psi} \subset C$.

In the case $\beta_{k} \equiv \beta, \beta \in \mathbb{R}$, the classes $C_{\bar{\beta}, p}^{\psi}$ are denoted by $C_{\beta, p}^{\psi}$.

For $\psi(k)=k^{-r}, r>0$, the classes $C_{\bar{\beta}, p}^{\psi} C_{\beta, p}^{\psi}$ are denoted by $W_{\bar{\beta}, p}^{r}$ and $W_{\beta, p}^{r}$, respectively. The $W_{\beta, p}^{r}$ are the well-known Weyl-Nagy classes (see, e.g., $[12,29,31,32]$ ). In other words $W_{\beta, p}^{r}, 1 \leq p \leq \infty$, are the classes of $2 \pi$-periodic functions $f$, representable as convolutions of the form

$$
f(x)=\frac{a_{0}}{2}+\frac{1}{\pi} \int_{-\pi}^{\pi} \varphi(x-t) B_{r, \beta}(t) d t, \quad a_{0} \in \mathbb{R},
$$

the Weyl-Nagy kernels $B_{r, \beta}(\cdot)$ of the form

$$
B_{r, \beta}(t)=\sum_{k=1}^{\infty} k^{-r} \cos \left(k t-\frac{\beta \pi}{2}\right), \quad r>0, \quad \beta \in \mathbb{R},
$$


with functions $\varphi \in B_{p}^{0}$. The function $\varphi$ in the formula (3) is called the Weyl-Nagy derivative of the function $f$ and is denoted by $f_{\beta}^{r}$.

If $r \in \mathbb{N}$ and $\beta=r$, then the functions $B_{r, \beta}(\cdot)$ of the form (4) are the well-known Bernoulli kernels and the corresponding classes $W_{\beta, p}^{r}$ coincide with the well-known classes $W_{p}^{r}$, which consist of $2 \pi$-periodic functions $f$ with absolutely continuous derivatives $f^{(k)}$ up to $(r-1)$ th order inclusive and such that $\left\|f^{(r)}\right\|_{p} \leq 1$. In addition, for almost everywhere $x \in \mathbb{R} \quad f^{(r)}(x)=f_{r}^{r}(x)=\varphi(x)$, where $\varphi$ is the function from (3).

For $\psi(k)=e^{-\alpha k^{r}}, \alpha>0, r>0$, the classes $C_{\bar{\beta}, p}^{\psi}$ are denoted by $C_{\bar{\beta}, p}^{\alpha, r}$. In the case of $r=1, \beta_{k} \equiv \beta, \beta \in \mathbb{R}$, and $p=\infty$ the sets $C_{\bar{\beta}, p}^{\alpha, r}$ are well-known classes of the Poisson integrals $C_{\beta, \infty}^{\alpha, 1}$ (see, e.g., [30-32]).

If $f \in C$ by $E_{n}(f)_{C}$ we denote the best uniform approximation of the function $f$ by elements of the subspace $\mathcal{T}_{2 n-1}$ of trigonometric polynomials $T_{n-1}$ of the order $n-1$ :

$$
T_{n-1}(x)=\frac{\alpha_{0}}{2}+\sum_{k=1}^{n-1}\left(\alpha_{k} \cos k x+\beta_{k} \sin k x\right), \quad \alpha_{k}, \beta_{k} \in \mathbb{R} .
$$

Let $\mathfrak{N}$ be the some functional class from the space $C(\mathfrak{N} \subset C)$. Then the quantity

$$
E_{n}(\mathfrak{N})_{C}=\sup _{f \in \mathfrak{N}} E_{n}(f)_{C}=\sup _{f \in \mathfrak{N}} \inf _{T_{n-1} \in \mathcal{T}_{2 n-1}}\left\|f(\cdot)-T_{n-1}(\cdot)\right\|_{C}
$$

is called the best uniform approximation of the class $\mathfrak{N}$ by elements of the subspace $\mathcal{T}_{2 n-1}$ of trigonometric polynomials $T_{n-1}$ of the order $n-1$.

At present, the exact values for the quantities of the form (5) are known for important functional classes $\mathfrak{N}$. In particular, thanks to the articles of Favard [7,8], Akhiezer and Krein [1], Nikol'skii [14], Dzyadyk $[4,5]$, Stechkin [29] and Sun [34] the exact values of the best uniform approximations of the Weyl-Nagy classes $W_{\beta, \infty}^{r}$ are found for arbitrary $r>0$ and $\beta \in \mathbb{R}$.

For the classes of the Poisson integrals $C_{\beta, \infty}^{\alpha, 1}$ the exact values of the form (5) are also known for all $\alpha>0$ and $\beta \in \mathbb{R}$ thanks to the articles of Krein [11], Bushanskij [3] and Shevaldin [28] (see also [2,32]).

The exact values of the best approximations $E_{n}(\mathfrak{N})_{C}$ were obtained in a number of other cases (see, e.g., [16-20,22,32]).

In the general case, the problem of finding of the exact values of the best uniform approximations of the classes $C_{\bar{\beta}, p}^{\psi}$ for $1 \leq p \leq \infty$ remains open, and therefore, the investigation of the asymptotic behavior of the quantities $E_{n}\left(C_{\bar{\beta}, p}^{\psi}\right)_{C}$ as $n \rightarrow \infty$ is relevant. 
In this paper we investigate the problem of finding of the asymptotic equalities for the quantities (5) as $n \rightarrow \infty$ in the case, when the classes $\mathfrak{N}$ are the classes $C_{\bar{\beta}, p}^{\psi}, 1 \leq p \leq \infty$, and the sequences $\psi(k)$ decrease to zero very rapidly, in particular, when

$$
\sum_{k=n+1}^{\infty} \psi(k)=o(1) \psi(n) .
$$

This work can be considered a continuation of the authors' research $[21,24,25]$, in which the asymptotics of the best uniform approximations of classes of the generalized $(\psi, \bar{\beta})$-integrals were investigated.

Note that in the case of $p=\infty$ the asymptotic equalities and even the exact values of the quantities $E_{n}\left(C_{\bar{\beta}, \infty}^{\psi}\right)_{C}$ are known under certain restrictions on $\psi(k)$ (see, e.g., [22,33]).

For a fixed $\mathfrak{N} \subset C$ denote by $\mathcal{E}_{n}(\mathfrak{N})_{C}$ the quantity

$$
\mathcal{E}_{n}(\mathfrak{N})_{C}=\sup _{f \in \mathfrak{N}}\left\|f(\cdot)-S_{n-1}(f ; \cdot)\right\|_{C},
$$

where $S_{n-1}(f ; \cdot)$ is the partial Fourier sum of order $n-1$ of the function $f$.

Since

$$
E_{n}(\mathfrak{N})_{C} \leq \mathcal{E}_{n}(\mathfrak{N})_{C}, \quad \mathfrak{N} \subset C,
$$

then the quantities (7) are naturally used for upper bounds for the best approximations of the classes $\mathfrak{N}$.

The problem of finding of the asymptotic equalities for the quantities of the form (7) as $n \rightarrow \infty$ is called the KolmogorovNikolskii problem for the Fourier sums. The KolmogorovNikolskii problem has a rich history. Reviews on the history of this problem can be found e.g. in the monographs [31,32].

For characteristics of the form (7) on the Weyl-Nagy classes $W_{\beta, \infty}^{r}$ $\left(\mathfrak{N}=W_{\beta, \infty}^{r}\right)$ the following asymptotic formula holds

$$
\mathcal{E}_{n}\left(W_{\beta, \infty}^{r}\right)_{C}=\frac{4}{\pi} \frac{\ln n}{n^{r}}+\mathcal{O}\left(\frac{1}{n^{r}}\right), \quad r>0, \quad \beta \in \mathbb{R} .
$$

For $r \in \mathbb{N}$ and $\beta=r$ this estimate was obtained by Kolmogorov [9], for arbitrary $r>0$ by Pinkevich [15] and Nikol'skii [13]. In the general case the estimate (9) follows from results of Efimov [6] and Telyakovskii [35].

In these works the parameters $r$ and $\beta$ of the Weyl-Nagy classes were assumed to be fixed, and the question about the dependence of the remainder term in the estimates (9) on these parameters was not 
considered. The character of the dependence on $r$ and $\beta$ of the remainder term in the estimate (9) was investigated by Sokolov, Selivanova, Natanson, Telyakovskii, Stechkin and other authors (see [26,30,36,37] and the references therein).

In the work of Stechkin [30] the asymptotic behavior of the quantities $\mathcal{E}_{n}\left(W_{\beta, \infty}^{r}\right)_{C}$ was completely investigated as $n \rightarrow \infty$ and $r \rightarrow \infty$. Namely, he proved that for arbitrary $r \geq 1$ and $\beta \in \mathbb{R}$ the following equality takes place

$$
\mathcal{E}_{n}\left(W_{\beta, \infty}^{r}\right)_{C}=\frac{1}{n^{r}}\left(\frac{8}{\pi^{2}} \mathbf{K}\left(e^{-r / n}\right)+\mathcal{O}(1) \frac{1}{r}\right),
$$

where

$$
\mathbf{K}(q)=\int_{0}^{\pi / 2} \frac{d t}{\sqrt{1-q^{2} \sin ^{2} t}}
$$

is a complete elliptic integral of the first kind, and $\mathcal{O}(1)$ is a quantity uniformly bounded with respect to $r, n$ and $\beta$.

In addition, Stechkin [30, Theorem 4] proved that for rapidly growing $r$ the remainder in the equality (10) can be improved. Namely, for arbitrary $r \geq n+1$ and $\beta \in \mathbb{R}$ the following formula holds

$$
\mathcal{E}_{n}\left(W_{\beta, \infty}^{r}\right)_{C}=\frac{1}{n^{r}}\left(\frac{4}{\pi}+\mathcal{O}(1)\left(1+\frac{1}{n}\right)^{-r}\right),
$$

where $\mathcal{O}(1)$ is a quantity uniformly bounded with respect to $r, n$ and $\beta$. If $r / n \rightarrow \infty$ then the estimate (12) becomes the asymptotic equality.

In the works of Telyakovskii $[36,37]$ it was shown that the second term in the formula (12) can be replaced by a smaller one, namely, we can write $\mathcal{O}(1)(1+2 / n)^{-r}$ instead of $\mathcal{O}(1)(1+1 / n)^{-r}$, and it s also the estimate (12) sharper by separating out the following terms of the asymptotics.

In the work of authors [26], in particular, the formula of Stechkin (12) was generalized on classes $W_{\bar{\beta}, p}^{r}$. Namely, it is proved that if $1 \leq$ $p \leq \infty, n \in \mathbb{N}$ and $\bar{\beta}=\left\{\beta_{k}\right\}_{k=1}^{\infty}$ is arbitrary sequence of real numbers, then for $r \geq n+1$ the following estimate holds

$$
\mathcal{E}_{n}\left(W_{\bar{\beta}, p}^{r}\right)_{C}=n^{-r}\left(\frac{\|\cos t\|_{p^{\prime}}}{\pi}+\mathcal{O}(1)\left(1+\frac{1}{n}\right)^{-r}\right), \quad \frac{1}{p}+\frac{1}{p^{\prime}}=1,
$$

where $\mathcal{O}(1)$ is a quantity uniformly bounded in all parameters.

For the classes of the generalized Poisson integrals $C_{\beta, p}^{\alpha, r}$ and for all fixed $\alpha>0, r>0, \beta \in \mathbb{R}$ and $1 \leq p \leq \infty$ the asymptotic equalities 
for the quantities (7) as $n \rightarrow \infty$ are known due to works of Nikol'skii [14], Stechkin [30], Stepanets [31,32], Telyakovskii [36,37], Serdyuk and Stepanyuk [27].

As for the classes $C_{\bar{\beta}, p}^{\psi}$, for the rapidly decreasing sequences $\psi(k)$ (in particular, when the condition (6) is satisfied) the asymptotics of the quantities $\mathcal{E}_{n}\left(C_{\bar{\beta}, p}^{\psi}\right)_{C}$ are known for all $1 \leq p \leq \infty$ and $\beta_{k} \in \mathbb{R}$ (see $[23,27,31,32,36,37])$. Therefore, the main goal of this work is focused on finding lower estimates for the best approximations $E_{n}\left(C_{\bar{\beta}, p}^{\psi}\right)_{C}, 1 \leq$ $p \leq \infty$.

In present paper we find two-sides estimates for the quantities $E_{n}\left(C_{\bar{\beta}, p}^{\psi}\right)_{C}$ and $\mathcal{E}_{n}\left(C_{\bar{\beta}, p}^{\psi}\right)_{C}$, from which, in particular, it follows that under condition $(6)$

$$
E_{n}\left(C_{\bar{\beta}, p}^{\psi}\right)_{C} \sim \mathcal{E}_{n}\left(C_{\bar{\beta}, p}^{\psi}\right)_{C} \sim \frac{\|\cos t\|_{p^{\prime}}}{\pi} \psi(n),
$$

where $1 \leq p \leq \infty, \quad \beta_{k} \in \mathbb{R}, \frac{1}{p}+\frac{1}{p^{\prime}}=1$, and $A(n) \sim B(n)$ as $n \rightarrow \infty$ means that $\lim _{n \rightarrow \infty} A(n) / B(n)=1$.

It should be noted that the asymptotic equality (14) is a manifestation of the high-smoothness effect, which occurs when the first harmonic in the remainder of the Fourier series after the $(n-1)$-th member of the generating kernels $\Psi_{\bar{\beta}}(t)$ of the form (2) is a dominant in estimating of the $L_{p^{\prime}}$-norm of the specified remainder of the series. When the sequences $\psi(k)$ decrease to zero not so fast, the equalities $E_{n}\left(C_{\bar{\beta}, p}^{\psi}\right)_{C}$ and $\mathcal{E}_{n}\left(C_{\bar{\beta}, p}^{\psi}\right)_{C}$ for $1 \leq p<\infty$ do not asymptotically coincide with each other and for $p=\infty$ can be different even in order.

Let $n \in \mathbb{N}$. In what follows, we will require that the sequence of modules of the Fourier coefficients of the generated kernel $\Psi_{\bar{\beta}}(t)$ satisfies the condition

$$
\sum_{k=n+1}^{\infty} \psi(k)<\psi(n) .
$$

Theorem 1. For arbitrary $\left\{\beta_{k}\right\}_{k=1}^{\infty}, \beta_{k} \in \mathbb{R}, 1<p \leq \infty, n \in \mathbb{N}$ and $\psi(k) \geq 0$, which satisfy the condition (15), the following inequality holds

$$
E_{n}\left(C_{\bar{\beta}, p}^{\psi}\right)_{C} \geq \frac{\|\cos t\|_{p^{\prime}}}{\pi}\left(\psi(n)-\sum_{k=n+1}^{\infty} \psi(k)\right),
$$

where $\frac{1}{p}+\frac{1}{p^{\prime}}=1$. 
Proof. Let us consider a function

$$
\varphi_{n, p}(t)=\|\cos t\|_{p^{\prime}}^{1-p^{\prime}}|\cos n t|^{p^{\prime}-1} \operatorname{sign} \cos n t .
$$

Since $\varphi_{n, p} \perp 1$ and for $1<p<\infty$

$$
\begin{gathered}
\left(\int_{-\pi}^{\pi}\left|\varphi_{n, p}(t)\right|^{p} d t\right)^{\frac{1}{p}}=\|\cos t\|_{p^{\prime}}^{1-p^{\prime}}\left(\int_{-\pi}^{\pi}|\cos n t|^{p\left(p^{\prime}-1\right)} d t\right)^{\frac{1}{p}}= \\
=\|\cos t\|_{p^{\prime}}^{1-p^{\prime}}\left(\int_{-\pi}^{\pi}|\cos n t|^{p^{\prime}} d t\right)^{\frac{1}{p}}= \\
=\|\cos t\|_{p^{\prime}}^{1-p^{\prime}}\left(\left(\int_{-\pi}^{\pi}|\cos n t|^{p^{\prime}} d t\right)^{\frac{1}{p^{\prime}}}\right)^{\frac{p^{\prime}}{p}}=\|\cos t\|_{p^{\prime}}^{1-p^{\prime}}\|\cos t\|_{p^{\prime}}^{\frac{p^{\prime}}{p}}=1,
\end{gathered}
$$

then $\left\|\varphi_{n, p}\right\|_{p}=1$ and therefore $\varphi_{n, p} \in B_{p}^{0}, 1<p<\infty$.

We also put $\varphi_{n, \infty}(t)=\operatorname{sign} \cos n t$. Obviously that $\varphi_{n, \infty} \in B_{\infty}^{0}$.

Let us consider a function

$$
f_{n, p, \bar{\beta}}(x)=\frac{1}{\pi} \int_{-\pi}^{\pi} \varphi_{n, p}(x-t) \Psi_{\bar{\beta}}(t) d t
$$

from the class $C_{\bar{\beta}, p}^{\psi}, 1<p \leq \infty$. We have

$$
\begin{gathered}
f_{n, p, \bar{\beta}}(x)=\frac{1}{\pi} \int_{-\pi}^{\pi} \varphi_{n, p}(x-t) \sum_{k=1}^{\infty} \psi(k) \cos \left(k t-\frac{\beta_{k} \pi}{2}\right) d t= \\
=\frac{1}{\pi} \int_{-\pi}^{\pi} \varphi_{n, p}(x-t) \sum_{k=1}^{n-1} \psi(k) \cos \left(k t-\frac{\beta_{k} \pi}{2}\right) d t+ \\
+\frac{1}{\pi} \int_{-\pi}^{\pi} \varphi_{n, p}(x-t) \sum_{k=n}^{\infty} \psi(k) \cos \left(k t-\frac{\beta_{k} \pi}{2}\right) d t .
\end{gathered}
$$

Since $\varphi_{n, p}(t)$ is the $\frac{2 \pi}{n}$-periodic function, then according to [10, Proposition 4.1.2] it is orthogonal to all trigonometric polynomials of order $n-1$. Therefore, from () we obtain

$$
f_{n, p, \bar{\beta}}(x)=\frac{1}{\pi} \int_{-\pi}^{\pi} \varphi_{n, p}(x-t) \sum_{k=n}^{\infty} \psi(k) \cos \left(k t-\frac{\beta_{k} \pi}{2}\right) d t=
$$




$$
\begin{gathered}
=\frac{1}{\pi} \int_{-\pi}^{\pi} \varphi_{n, p}(t) \psi(n) \cos \left(n(x-t)-\frac{\beta_{n} \pi}{2}\right)+ \\
+\frac{1}{\pi} \int_{-\pi}^{\pi} \varphi_{n, p}(x-t) \sum_{k=n+1}^{\infty} \psi(k) \cos \left(k t-\frac{\beta_{k} \pi}{2}\right) d t=: F_{1}(x)+F_{2}(x) .
\end{gathered}
$$

By virtue of Hölder's inequality and the fact that $\varphi_{n, p} \in B_{p}^{0}, 1<p \leq$ $\infty$, we obtain the estimate

$$
\begin{array}{r}
\left\|F_{2}\right\|_{C} \leq \frac{1}{\pi}\left\|\varphi_{n, p}\right\|_{p}\left\|\sum_{k=n+1}^{\infty} \psi(k) \cos \left(k t-\frac{\beta_{k} \pi}{2}\right)\right\|_{p^{\prime}}= \\
=\frac{1}{\pi}\left\|\sum_{k=n+1}^{\infty} \psi(k) \cos \left(k t-\frac{\beta_{k} \pi}{2}\right)\right\|_{p^{\prime}} \leq \frac{\|\cos t\|_{p^{\prime}}}{\pi} \sum_{k=n+1}^{\infty} \psi(k) .
\end{array}
$$

Taking into account that

$$
\begin{gathered}
F_{1}(x)= \\
=\frac{1}{\pi} \int_{-\pi}^{\pi}\|\cos t\|_{p^{\prime}}^{1-p^{\prime}}|\cos n t|^{p^{\prime}-1} \operatorname{sign} \cos n t \psi(n) \cos \left(n(x-t)-\frac{\beta_{n} \pi}{2}\right) d t= \\
=\frac{\|\cos t\|_{p^{\prime}}^{1-p^{\prime}}}{\pi} \psi(n) \int_{-\pi}^{\pi}|\cos n t|^{p^{\prime}-1} \operatorname{sign} \cos n t \times \\
\times\left(\cos \left(n x-\frac{\beta_{n} \pi}{2}\right) \cos n t+\sin \left(n x-\frac{\beta_{n} \pi}{2}\right) \sin n t\right) d t
\end{gathered}
$$

we consider on the period $\left[\frac{\beta_{n} \pi}{2 n}, 2 \pi+\frac{\beta_{n} \pi}{2 n}\right)$ the set of $2 n$ points $\frac{\beta_{n} \pi}{2 n}=$ $x_{0}<x_{1}<\ldots<x_{2 n-1}<2 \pi+\frac{\beta_{n} \pi}{2 n}$ of the form

$$
x_{m}=\frac{\beta_{n} \pi}{2 n}+\frac{m \pi}{n}, \quad m=\overline{0,2 n-1} .
$$

Let us show that

$$
F_{1}\left(x_{m}\right)=\frac{(-1)^{m}\|\cos t\|_{p^{\prime}}}{\pi} \psi(n), \quad m=\overline{0,2 n-1} .
$$

Indeed, as for any $t \in[-\pi, \pi)$

$$
|\cos n t|^{p^{\prime}-1} \operatorname{sign} \cos n t \cos \left(n\left(x_{m}-t\right)-\frac{\beta_{n} \pi}{2}\right)=
$$




$$
\begin{aligned}
& =|\cos n t|^{p^{\prime}-1} \operatorname{sign} \cos n t \cos \left(\frac{\beta_{n} \pi}{2}+m \pi-n t-\frac{\beta_{n} \pi}{2}\right)= \\
& =(-1)^{m}|\cos n t|^{p^{\prime}-1} \operatorname{sign} \cos n t \cos n t=(-1)^{m}|\cos n t|^{p^{\prime}},
\end{aligned}
$$

then

$$
\begin{gathered}
F_{1}\left(x_{m}\right)= \\
=\frac{\|\cos t\|_{p^{\prime}}^{1-p^{\prime}}}{\pi} \psi(n) \int_{-\pi}^{\pi}|\cos n t|^{p^{\prime}-1} \operatorname{sign} \cos n t \cos \left(n\left(x_{m}-t\right)-\frac{\beta_{n} \pi}{2}\right) d t= \\
=\frac{\|\cos t\|_{p^{\prime}}^{1-p^{\prime}}}{\pi} \psi(n)(-1)^{m} \int_{-\pi}^{\pi}|\cos n t|^{p^{\prime}} d t=\frac{(-1)^{m}\|\cos t\|_{p^{\prime}}}{\pi} \psi(n) .
\end{gathered}
$$

From (15), () and (22) it follows that

$$
\left|F_{1}\left(x_{m}\right)\right|=\frac{\|\cos t\|_{p^{\prime}}}{\pi} \psi(n)>\frac{\|\cos t\|_{p^{\prime}}}{\pi} \sum_{k=n+1}^{\infty} \psi(k) \geq\left\|F_{2}\right\|_{C} \geq\left|F_{2}\left(x_{m}\right)\right|,
$$

and hence for $f_{n, p, \bar{\beta}}\left(x_{m}\right)=F_{1}\left(x_{m}\right)+F_{2}\left(x_{m}\right)$ the following relations hold

$$
\operatorname{sign} f_{n, p, \bar{\beta}}\left(x_{m}\right)=\operatorname{sign} F_{1}\left(x_{m}\right)=(-1)^{m}, \quad m=0,1, \ldots, 2 n-1,
$$

and

$$
\begin{aligned}
\left|f_{n, p, \bar{\beta}}\left(x_{m}\right)\right| & \geq\left|F_{1}\left(x_{m}\right)\right|-\left|F_{2}\left(x_{m}\right)\right| \geq\left|F_{1}\left(x_{m}\right)\right|-\left\|F_{2}\right\|_{C} \geq \\
& \geq \frac{\|\cos t\|_{p^{\prime}}}{\pi}\left(\psi(n)-\sum_{k=n+1}^{\infty} \psi(k)\right) .
\end{aligned}
$$

Then by virtue of Valle Poussin's theorem (see, i.e., [31, Theorem 6.2.2]) $E_{n}\left(f_{n, p, \bar{\beta}}\right)_{C} \geq \min _{m=0,1, \ldots, 2 n-1}\left|f_{n, p, \bar{\beta}}\left(x_{m}\right)\right| \geq \frac{\|\cos t\|_{p^{\prime}}}{\pi}\left(\psi(n)-\sum_{k=n+1}^{\infty} \psi(k)\right)$.

Theorem 2. For arbitrary $\left\{\beta_{k}\right\}_{k=1}^{\infty}, \beta_{k} \in \mathbb{R}, n \in \mathbb{N}$ and $\psi(k) \geq 0$, which satisfy the condition (15), the following inequality holds

$$
E_{n}\left(C_{\bar{\beta}, 1}^{\psi}\right)_{C} \geq \frac{1}{\pi}\left(\psi(n)-\sum_{k=n+1}^{\infty} \psi(k)\right)
$$


Proof. Let us consider a function

$$
\varphi_{n, 1}(\delta, t)=\left\{\begin{array}{cl}
\frac{(-1)^{m}}{2 \delta}, & t \in\left(\frac{m \pi}{n}-\frac{\delta}{2 n}, \frac{m \pi}{n}+\frac{\delta}{2 n}\right), m \in \mathbb{Z}, \\
0, & t \in \mathbb{R} \backslash \underset{m \in \mathbb{Z}}{\cup}\left(\frac{m \pi}{n}-\frac{\delta}{2 n}, \frac{m \pi}{n}+\frac{\delta}{2 n}\right), m \in \mathbb{Z},
\end{array}\right.
$$

where $0<\delta<\frac{\pi}{2}$.

According to the definition

$$
\operatorname{sign} \varphi_{n, 1}(\delta, t)=\operatorname{sign} \cos n t, \quad t \in\left(\frac{m \pi}{n}-\frac{\delta}{2 n}, \frac{m \pi}{n}+\frac{\delta}{2 n}\right), m \in \mathbb{Z},
$$

and the function $\varphi_{n, 1}(\delta, t)$ is $\frac{2 \pi}{n}$-periodic function. Besides

$$
\left\|\varphi_{n, 1}(\delta, \cdot)\right\|_{1}=\sum_{m=0}^{2 n-1} \frac{1}{2 \delta} \frac{2 \delta}{2 n}=1,
$$

that is for any $0<\delta<\frac{\pi}{2} \varphi_{n, 1}(\delta, \cdot) \in B_{1}^{0}$.

Let us consider a function

$$
f_{n, 1, \bar{\beta}}(\delta, x)=\frac{1}{\pi} \int_{-\pi}^{\pi} \varphi_{n, 1}(\delta, x-t) \Psi_{\bar{\beta}}(t) d t
$$

from the class $C_{\bar{\beta}, 1}^{\psi}$. Since $\varphi_{n, 1}(\delta, t) \perp \sum_{k=1}^{n-1} \psi(k) \cos \left(k t-\frac{\beta_{k} \pi}{2}\right)$, then

$$
f_{n, 1, \bar{\beta}}(\delta, x)=F_{1}(\delta, x)+F_{2}(\delta, x)
$$

where

$$
\begin{gathered}
F_{1}(\delta, x)=\frac{1}{\pi} \int_{-\pi}^{\pi} \varphi_{n, 1}(\delta, x-t) \psi(n) \cos \left(n t-\frac{\beta_{n} \pi}{2}\right) d t= \\
=\frac{1}{\pi} \psi(n) \int_{-\pi}^{\pi} \varphi_{n, 1}(\delta, t) \cos \left(n(x-t)-\frac{\beta_{n} \pi}{2}\right) d t \\
F_{2}(\delta, x)=\frac{1}{\pi} \int_{-\pi}^{\pi} \varphi_{n, 1}(\delta, x-t) \sum_{k=n+1}^{\infty} \psi(k) \cos \left(k t-\frac{\beta_{k} \pi}{2}\right) d t
\end{gathered}
$$


By virtue of Hölder's inequality and the equality (28)

$$
\begin{gathered}
\left\|F_{2}(\delta, \cdot)\right\|_{C} \leq \frac{1}{\pi}\left\|\varphi_{n, 1}(\delta, \cdot)\right\|_{1}\left\|\sum_{k=n+1}^{\infty} \psi(k) \cos \left(k t-\frac{\beta_{k} \pi}{2}\right)\right\|_{\infty} \leq \\
\leq \frac{1}{\pi} \sum_{k=n+1}^{\infty} \psi(k) .
\end{gathered}
$$

Let us consider on the segment $\left[\frac{\beta_{n} \pi}{2 n}, 2 \pi+\frac{\beta_{n} \pi}{2 n}\right)$ the following point set

$$
x_{m}=\frac{\beta_{n} \pi}{2 n}+\frac{m \pi}{n}, \quad m=\overline{0,2 n-1} .
$$

Let us show that

$$
F_{1}\left(\delta, x_{m}\right)=\frac{(-1)^{m}}{\pi} \frac{2}{\delta} \sin \frac{\delta}{2} \psi(n), \quad m=\overline{0,2 n-1} .
$$

Indeed, since

$$
\begin{gathered}
\varphi_{n, 1}(\delta, t) \cos \left(n\left(x_{m}-t\right)-\frac{\beta_{n} \pi}{2}\right)= \\
=\varphi_{n, 1}(\delta, t) \cos \left(n\left(\frac{\beta_{n} \pi}{2 n}+\frac{m \pi}{n}-t\right)-\frac{\beta_{n} \pi}{2}\right)= \\
=\varphi_{n, 1}(\delta, t) \cos (m \pi-n t)=(-1)^{m} \varphi_{n, 1}(\delta, t) \cos n t,
\end{gathered}
$$

Then, in view of (27), (28) and (),

$$
\begin{gathered}
F_{1}\left(x_{m}\right)=\frac{1}{\pi} \psi(n) \int_{-\pi}^{\pi}(-1)^{m} \varphi_{n, 1}(\delta, t) \cos n t d t= \\
=\frac{(-1)^{m}}{\pi} \psi(n) \sum_{m=0}^{2 n-1} \int_{-\frac{\pi}{2 n}+\frac{m \pi}{2 n}}^{\frac{\pi}{2 n}+\frac{m \pi}{2 n}} \varphi_{n, 1}(\delta, t) \cos n t d t= \\
=\frac{(-1)^{m}}{\pi} \psi(n) \sum_{m=0}^{2 n-1} \int_{-\frac{\delta}{2 n}+\frac{m \pi}{2 n}}^{\frac{\delta}{2 n}+\frac{m \pi}{2 n}} \frac{(-1)^{m}}{2 \delta} \cos n t d t= \\
=\frac{(-1)^{m}}{\pi} \psi(n) \sum_{m=0}^{2 n-1} \frac{1}{2 \delta} \int_{-\frac{\delta}{2 n}}^{\frac{\delta}{2 n}} \cos n t d t=
\end{gathered}
$$




$$
=\frac{(-1)^{m}}{\pi} \psi(n) \frac{2 n}{2 \delta n} \int_{-\frac{\delta}{2}}^{\frac{\delta}{2}} \cos t d t=\frac{(-1)^{m}}{\pi} \psi(n) \frac{2}{\delta} \sin \frac{\delta}{2} .
$$

Choose $\delta$ so small that

$$
\frac{2}{\delta} \sin \frac{\delta}{2} \psi(n)>\sum_{k=n+1}^{\infty} \psi(k)
$$

For such $\delta$, taking into account (), (33) and (34),

$$
\left|F_{1}\left(\delta, x_{m}\right)\right|=\frac{2}{\delta} \sin \frac{\delta}{2} \psi(n)>\frac{1}{\pi} \sum_{k=n+1}^{\infty} \psi(k) \geq\left\|F_{2}(\delta, \cdot)\right\|_{C} \geq\left|F_{2}\left(\delta, x_{m}\right)\right|,
$$

and hence for $f_{n, p, \bar{\beta}}\left(\delta, x_{m}\right)=F_{1}\left(\delta, x_{m}\right)+F_{2}\left(\delta, x_{m}\right)$ the following relations hold

$$
\operatorname{sign} f_{n, p, \bar{\beta}}\left(\delta, x_{m}\right)=\operatorname{sign} F_{1}\left(\delta, x_{m}\right)=(-1)^{m}, \quad m=0,1, \ldots, 2 n-1,
$$

and

$$
\begin{gathered}
\left|f_{n, p, \bar{\beta}}\left(\delta, x_{m}\right)\right| \geq\left|F_{1}\left(\delta, x_{m}\right)\right|-\left|F_{2}\left(\delta, x_{m}\right)\right| \geq\left|F_{1}\left(\delta, x_{m}\right)\right|-\left\|F_{2}(\delta, \cdot)\right\|_{C} \geq \\
\geq \frac{1}{\pi}\left(\frac{2}{\delta} \sin \frac{\delta}{2} \psi(n)-\sum_{k=n+1}^{\infty} \psi(k)\right) .
\end{gathered}
$$

Then by virtue of Valle Poussin's theorem (see, i.e., [31, Theorem 6.2.2])

$$
\begin{gathered}
E_{n}\left(f_{n, p, \bar{\beta}}(\delta, \cdot)\right)_{C} \geq \min _{m=0,1, \ldots, 2 n-1}\left|f_{n, p, \bar{\beta}}\left(\delta, x_{m}\right)\right| \geq \\
\geq \frac{1}{\pi}\left(\frac{2}{\delta} \sin \frac{\delta}{2} \psi(n)-\sum_{k=n+1}^{\infty} \psi(k)\right),
\end{gathered}
$$

where $\delta$ satisfy the condition (34), and therefore, by virtue of the belonging $f_{n, p, \bar{\beta}}(\delta, \cdot) \in C_{\bar{\beta}, 1}^{\psi}$, the following inequality is true

$$
E_{n}\left(C_{\bar{\beta}, 1}^{\psi}\right)_{C} \geq \frac{1}{\pi}\left(\frac{2}{\delta} \sin \frac{\delta}{2} \psi(n)-\sum_{k=n+1}^{\infty} \psi(k)\right) .
$$

Taking the limit as $\delta \rightarrow 0$ in the inequality (38), we obtain (25).

The main statement of the work is the following theorem. 
Theorem 3. For arbitrary $\left\{\beta_{k}\right\}_{k=1}^{\infty}, \beta_{k} \in \mathbb{R}, 1<p \leq \infty, n \in \mathbb{N}$ and $\psi(k) \geq 0$, which satisfy the condition (15), the following inequalities hold

$$
\begin{aligned}
& \frac{\|\cos t\|_{p^{\prime}}}{\pi}\left(\psi(n)-\sum_{k=n+1}^{\infty} \psi(k)\right) \leq \\
& \leq E_{n}\left(C_{\bar{\beta}, p}^{\psi}\right)_{C} \leq \mathcal{E}_{n}\left(C_{\bar{\beta}, p}^{\psi}\right)_{C} \leq \\
& \leq \frac{\|\cos t\|_{p^{\prime}}}{\pi}\left(\psi(n)+\sum_{k=n+1}^{\infty} \psi(k)\right),
\end{aligned}
$$

where $\frac{1}{p}+\frac{1}{p^{\prime}}=1$.

If $\psi(k)$ satisfies the condition (6), then the following asymptotic equalities hold

$$
\left.\begin{array}{l}
\mathcal{E}_{n}\left(C_{\bar{\beta}, p}^{\psi}\right)_{C} \\
E_{n}\left(C_{\bar{\beta}, p}^{\psi}\right)_{C}
\end{array}\right\}=\frac{\|\cos t\|_{p^{\prime}}}{\pi} \psi(n)+\mathcal{O}(1) \sum_{k=n+1}^{\infty} \psi(k),
$$

where $\mathcal{O}(1)$ are the quantities uniformly bounded in all parameters.

Note that the asymptotic equality (40) for the quantities $\mathcal{E}_{n}\left(C_{\bar{\beta}, p}^{\psi}\right)_{C}$ is established in paper [23].

Proof. Taking into account Theorems 1 and 2, it suffices to verify the validity of the last inequality in (39).

By virtue of Hölder's inequality for arbitrary $f \in C_{\bar{\beta}, p}^{\psi}, 1 \leq p \leq \infty$,

$$
\begin{aligned}
& \left\|f-S_{n-1}(f)\right\|_{C} \leq \frac{1}{\pi}\|\varphi\|_{p}\left\|\sum_{k=n}^{\infty} \psi(k) \cos \left(n t-\frac{\beta_{k} \pi}{2}\right)\right\|_{p^{\prime}} \leq \\
& \leq \frac{1}{\pi} \sum_{k=n}^{\infty} \psi(k)\left\|\cos \left(n t-\frac{\beta_{k} \pi}{2}\right)\right\|_{p^{\prime}}=\frac{\|\cos t\|_{p^{\prime}}}{\pi} \sum_{k=n}^{\infty} \psi(k) .
\end{aligned}
$$

Thus,

$$
\mathcal{E}_{n}\left(C_{\bar{\beta}, p}^{\psi}\right)_{C} \leq \frac{\|\cos t\|_{p^{\prime}}}{\pi} \sum_{k=n}^{\infty} \psi(k)
$$

Inequalities (39) follow from (16), (25) and (41).

To verify the validity of the asymptotic equalities (40) just go to the limit as $n \rightarrow \infty$ in (39) and take into account the condition (6). 
Note that the condition (6) is satisfied if $\psi(k)$ satisfies the condition $D_{0}$ :

$$
\lim _{k \rightarrow \infty} \frac{\psi(k+1)}{\psi(k)}=0
$$

We give the corollaries of Theorem 3 in some important special cases.

Theorem 4. Let $1 \leq p \leq \infty, r>1, n \in \mathbb{N}$ and $\left\{\beta_{k}\right\}_{k=1}^{\infty}$ be an arbitrary sequence of real numbers. Then for $r \geq n+1$ such that

$$
\left(1+\frac{1}{n}\right)^{-r}<\left(2+\frac{1}{n}\right)^{-1}
$$

the following inequalities hold

$$
\begin{aligned}
& \frac{\|\cos t\|_{p^{\prime}}}{\pi} n^{-r}\left(1-\frac{2+\frac{1}{n}}{\left(1+\frac{1}{n}\right)^{r}}\right) \leq \\
& \leq E_{n}\left(W_{\bar{\beta}, p}^{r}\right)_{C} \leq \mathcal{E}_{n}\left(W_{\bar{\beta}, p}^{r}\right)_{C} \leq \\
& \leq \frac{\|\cos t\|_{p^{\prime}}}{\pi} n^{-r}\left(1+\frac{2+\frac{1}{n}}{\left(1+\frac{1}{n}\right)^{r}}\right),
\end{aligned}
$$

where $\frac{1}{p}+\frac{1}{p^{\prime}}=1$.

Proof. Put $\psi(k)=k^{-r}, r>1$, and make sure that the condition (15) is folowed from the inequality (43).

Since for arbitrary $n \in \mathbb{N}$ and $r \geq n+1$

$$
\begin{aligned}
& \sum_{k=n+1}^{\infty} \frac{1}{k^{r}}<\frac{1}{(n+1)^{r}}+\int_{n+1}^{\infty} \frac{d t}{t^{r}}=\frac{1}{(n+1)^{r}}+\frac{1}{(r-1)(n+1)^{r-1}}= \\
= & \frac{1}{(n+1)^{r}} \frac{r+n}{r-1} \leq \frac{1}{n^{r}} \frac{1}{\left(1+\frac{1}{n}\right)^{r}} \frac{2 r-1}{r-1} \leq \frac{1}{n^{r}} \frac{1}{\left(1+\frac{1}{n}\right)^{r}}\left(2+\frac{1}{n}\right),
\end{aligned}
$$

then under the condition (43) we obtain

$$
\sum_{k=n+1}^{\infty} \frac{1}{k^{r}}<\frac{1}{n^{r}} .
$$

Then, applying Theorem 3 for $\psi(k)=k^{-r}$, from (39) and () we obtain (44). 
Note that if the condition

$$
\lim _{n \rightarrow \infty} \frac{r}{n}=\infty
$$

is satisfied, then the condition (43) is also satisfied for sufficiently large $n$, because

$$
\left(1+\frac{1}{n}\right)^{-r}=\left(\left(1+\frac{1}{n}\right)^{n+1}\right)^{-\frac{r}{n+1}} \leq e^{-\frac{r}{n+1}} \rightarrow 0, \quad n \rightarrow \infty .
$$

And therefore

$$
\frac{2+\frac{1}{n}}{\left(1+\frac{1}{n}\right)^{r}} \rightarrow 0 \quad n \rightarrow \infty
$$

Taking the limit as $n \rightarrow \infty$ in the inequalities (44), we obtain asymptotic equalities for the quantities $E_{n}\left(W_{\bar{\beta}, p}^{r}\right)_{C}$ and $\mathcal{E}_{n}\left(W_{\bar{\beta}, p}^{r}\right)_{C}$.

Theorem 5. Let $1 \leq p \leq \infty, r>1, n \in \mathbb{N}$ and $\left\{\beta_{k}\right\}_{k=1}^{\infty}$ be an arbitrary sequence of real numbers. If the condition (46) is satisfied, then the following asymptotic equalities hold

$$
\left.\begin{array}{l}
\mathcal{E}_{n}\left(W_{\bar{\beta}, p}^{r}\right)_{C} \\
E_{n}\left(W_{\bar{\beta}, p}^{r}\right)_{C}
\end{array}\right\}=\frac{1}{n^{r}}\left(\frac{\|\cos t\|_{p^{\prime}}}{\pi}+\mathcal{O}(1)\left(1+\frac{1}{n}\right)^{-r}\right),
$$

where $\frac{1}{p}+\frac{1}{p^{\prime}}=1$ and $\mathcal{O}(1)$ are the quantities uniformly bounded in all parameters.

The asymptotic equality (47) for the quantities $\mathcal{E}_{n}\left(C_{\bar{\beta}, p}^{\psi}\right)_{C}$ is established in [26].

As it showed in [31, P. 163-164]

$$
\sum_{k=n+1}^{\infty} e^{-\alpha k^{r}}<e^{-\alpha n^{r}}\left(1+\frac{1}{\alpha r n^{r-1}}\right) e^{-\alpha r n^{r-1}}, \quad r>1, \alpha>0, n \in \mathbb{N} .
$$

So, if the condition

$$
\left(1+\frac{1}{\alpha r n^{r-1}}\right) e^{-\alpha r n^{r-1}}<1, \quad r>1, \alpha>0,
$$

is satysfied, then

$$
\sum_{k=n+1}^{\infty} e^{-\alpha k^{r}}<e^{-\alpha n^{r}}
$$

and therefore from Theorem 3 for the classes $C_{\bar{\beta}, p}^{\alpha, r}$ we obtain the following statement. 
Theorem 6. Let $1 \leq p \leq \infty, r>1, \alpha>0, n \in \mathbb{N}$ and $\left\{\beta_{k}\right\}_{k=1}^{\infty}$ be an arbitrary sequence of real numbers. If the inequality (49) is satisfied, then the following relations hold

$$
\begin{aligned}
& \frac{\|\cos t\|_{p^{\prime}}}{\pi} e^{-\alpha n^{r}}\left(1-\left(1+\frac{1}{\alpha r n^{r-1}}\right) e^{-\alpha r n^{r-1}}\right) \leq E_{n}\left(C_{\bar{\beta}, p}^{\alpha, r}\right)_{C} \leq \\
& \leq \mathcal{E}_{n}\left(C_{\bar{\beta}, p}^{\alpha, r}\right)_{C} \leq \frac{\|\cos t\|_{p^{\prime}}}{\pi} e^{-\alpha n^{r}}\left(1+\left(1+\frac{1}{\alpha r n^{r-1}}\right) e^{-\alpha r n^{r-1}}\right),
\end{aligned}
$$

where $\frac{1}{p}+\frac{1}{p^{\prime}}=1$.

As $n \rightarrow \infty$ the following asymptotic equalities hold

$$
\left.\begin{array}{l}
\mathcal{E}_{n}\left(C_{\bar{\beta}, p}^{\alpha, r}\right)_{C} \\
E_{n}\left(C_{\bar{\beta}, p}^{\alpha, r}\right)_{C}
\end{array}\right\}=e^{-\alpha n^{r}}\left(\frac{\|\cos t\|_{p^{\prime}}}{\pi}+\mathcal{O}(1)\left(1+\left(1+\frac{1}{\alpha r n^{r-1}}\right) e^{-\alpha r n^{r-1}}\right)\right)
$$

where $\mathcal{O}(1)$ are the quantities uniformly bounded in all parameters.

Note that for $p=\infty$ the asymptotic equality (50) for the quantities $\mathcal{E}_{n}\left(C_{\bar{\beta}, p}^{\alpha, r}\right)_{C}$ was obtained by Stepanets [31] and for $1 \leq p<\infty$ in [23].

\section{References}

[1] N.I. Akhiezer, M. G. Krein, On the best approximation of differentiable periodic functions by trigonometric polynomials // Dokl. Akad. Nauk SSSR, 15, No. 3 (1937), 107112.

[2] N. A. Baraboshkina, L-approximation of a linear combination of the Poisson kernel and its conjugate kernel by trigonometric polynomials // Proc. Steklov Inst. Math., 273, 1 (2011), 5967.

[3] A.V. Bushanskii, Best harmonic approximation in the mean of certain functions // Studies in the theory of approximation of functions and their applications, Akad. Nauk Ukrain. SSR, Inst. Mat., Kiev, 1978, 2937.

[4] V.K. Dzyadyk, On the best approximation on the classes of periodic functions determined by kernels which are integrals of absolutely monotone functions // Izv. Akad. Nauk SSSR, Ser. Mat., 23, No. 6 (1959), 933950.

[5] V. K. Dzyadyk, Best approximation on classes of periodic functions defined by integrals of linear combinations of absolutely monotonous kernels // Mat. Zametki,16, No. 5 (1974), 691701. 
[6] A. V. Efimov, Approximation of continuous periodic functions by Fourier sums // Izv. Akad. Nauk SSSR, Ser. Mat., 24 (1960), 243296.

[7] J. Favard, Sur l'approximation des fonctions périodiques par des polynomes trigonométriques // C.R.Acad. Sci., 203 (1936), 11221124 .

[8] J. Favard, Sur les meilleurs procédes d'approximations de certains classes de fontions par des polynomes trigonometriques // Bull. de Sci. Math., 61 (1937), 209-224, 243 - 256.

[9] A. N. Kolmogorov, On the order of the remainders of the Fourier series of differentiable functions // A.N. Kolmogorov, Selected Works. Mathematics and Mechanics, Nauka, Moscow (1985), 179185.

[10] N.P. Korneichuk, Exact constants in approximation theory, Encyclopedia of Mathematics and its Applications, 38. Cambridge University Press, Cambridge, 1991, xii+452 pp.

[11] M. G. Krein, The theory of best approximation of periodic functions // Dokl. Akad. Nauk SSSR, 18, Nos. 45 (1938), 245249.

[12] B. Sz.-Nagy, Über gewisse Extremalfragen bei transformierten trigonometrischen Entwicklungen. 1. Periodischer Fall // Ber. Math.-Phys. Kl. Akad. Wiss., Leipzig, 90 (1938), 103134.

[13] S. M. Nikol'skii, An asymptotic estimation of the remainder under approximation by Fourier sums // Dokl. Akad. Nauk SSSR, 32 (1941), 386389.

[14] S. M. Nikol'skii, Approximation of functions in the mean by trigonometric polynomials // Izv. Akad. Nauk SSSR, Ser. Mat., 10 (1946), 207256 .

[15] V. T. Pinkevich, On the order of the remainders of the Fourier series of functions differentiable in the sense of Weyl // Izv. Akad. Nauk SSSR, Ser. Mat., 4 (1940), 521528.

[16] A. Pinkus, n-widths in approximation theory, Springer-Verlag, Berlin, 1985. $\mathrm{x}+291$.

[17] A.S. Serdyuk, On the best approximation of classes of convolutions of periodic functions by trigonometric polynomials // Ukrainian Math. J. 47 (1995), no. 9, 14351440. 
[18] A.S. Serdyuk, Estimates for the widths and best approximations of classes of convolutions of periodic functions // Fourier series: theory and applications (Kamenets-Podol'skii, 1997), Pr. Inst. Mat. Nats. Akad. Nauk Ukr. Mat. Zastos., 20 (1998), 286299.

[19] A.S. Serdyuk, Widths and best approximations for classes of convolutions of periodic functions // Ukrainian Math. J. 51, no. 5 (1999), 748763 .

[20] A.S. Serdyuk, On best approximation in classes of convolutions of periodic functions // Theory of the approximation of functions and related problems, Pr. Inst. Mat. Nats. Akad. Nauk Ukr. Mat. Zastos., 35 (2002), 172194.

[21] A.S. Serdyuk, On one linear method of approximation of periodic functions // Zb. Pr. Inst. Mat. NAN Ukr. 1, No. 1 (2004), 294-336.

[22] A.S. Serdyuk, Best approximations and widths of classes of convolutions of periodic functions of high smoothness //Ukrainian Math. J. 57 (2005), no. 7, 11201148.

[23] A.S. Serdyuk, Approximation of classes of analytic functions by Fourier sums in the uniform metric // Ukrainian Math. J. 57 (2005), no. 8, 12751296.

[24] A.S. Serdyuk, I.V. Sokolenko, Asymptotic behavior of best approximations of classes of Poisson integrals of functions from $H_{\omega} / /$ Journal of Approximation Theory, 163, 11 (2011), 1692-1706.

[25] A.S. Serdyuk, I.V. Sokolenko, Asymptotic equalities for best approximations for classes of infinitely differentiable functions defined by the modulus of continuity // Math. Notes 99 (2016), no. 5-6, 901915.

[26] A.S. Serdyuk, I.V. Sokolenko, Approximation by Fourier sums in classes of differentiable functions with high exponents of smoothness // Methods of Functional Analysis and Topology. Vol. 25 (2019), $4,381-387$.

[27] A.S. Serdyuk, T.A. Stepanyuk, Uniform Approximations by Fourier Sums in Classes of Generalized Poisson Integrals // Analysis Math., 45 (1) (2019), 201236.

[28] V.T. Shevaldin, Widths of classes of convolutions with Poisson kernel // Math. Notes, 51:6 (1992), 611617. 
[29] S. B. Stechkin, On the best approximation of certain classes of periodic functions by trigonometric polynomials // Izv. Akad. Nauk SSSR, Ser. Mat., 20, No. 6 (1956), 643648.

[30] S. B. Stechkin, An estimation of the remainders of the Fourier series of differentiable functions // Tr. Mat. Inst. Akad. Nauk SSSR, 145 (1980), 126151.

[31] A. I. Stepanets, Classification and Approximation of Periodic Functions, Kluwer Academic Publishers, Dordrecht, 1995.

[32] A.I. Stepanets, Methods of Approximation Theory, Utrecht: VSP (2005).

[33] A.I. Stepanets, A.S. Serdyuk, Approximation by Fourier sums and best approximations on classes of analytic functions // Ukrainian Math. J. 52 (2000), no. 3, 433456.

[34] Sun Yong-sheng, On the best approximation of periodic differentiable functions by trigonometric polynomials. II // Izv. Akad. Nauk SSSR Ser. Mat., 25:1 (1961), 143152.

[35] S. A. Telyakovskii, On the norms of trigonometric polynomials and approximation of differentiable functions by the linear means of their Fourier series // Tr. Mat. Inst. Akad. Nauk SSSR, 62 (1961), 6197.

[36] S.A. Telyakovskii, Approximation by Fourier sums of functions of high smoothness // Ukrainian Math. J. 41 (1989), no. 4, 444451.

[37] S.A. Telyakovskii, On approximation by Fourier sums of differentiable functions of high smoothness // Proc. Steklov Inst. Math. 1994, 1 (198), 183201.

\section{CONTACT INFORMATION}

A.S. Serdyuk

01024, Ukraine, Kiev-4, 3, Tereschenkivska st. serdyuk@imath.kiev.ua

I.V. Sokolenko 01024, Ukraine, Kiev-4, 3, Tereschenkivska st. sokol@imath.kiev.ua 